\title{
HIV I AIDS Epidemic in the Democratic Republic of the Congo: Current Level of Key Indicators and Projection by 2030
}

\author{
Jack Hyyombo Tambwe Kokolomami*, Patrick Kalambayi Kayembe \\ Department of Epidemiology and Biostatistics, Kinshasa School of Public Health, University of Kinshasa, Kinshasa, the Democratic \\ Republic of Congo
}

Email address:

pajack70@yahoo.fr (J. H. T. Kokolomami)

${ }^{*}$ Corresponding author

\section{To cite this article:}

Jack Hyyombo Tambwe Kokolomami, Patrick Kalambayi Kayembe. HIV / AIDS Epidemic in the Democratic Republic of the Congo: Current Level of Key Indicators and Projection by 2030. Central African Journal of Public Health. Vol. 4, No. 3, 2018 , pp. 86-94. doi: 10.11648/j.cajph.20180403.16

Received: July 4, 2018; Accepted: July 17, 2018; Published: August 14, 2018

\begin{abstract}
Since 2015, the Democratic Republic of Congo has subscribed to the global goal of eliminating HIV / AIDS as a major public health problem by 2030 . However, there is a lack of evidence on the current level of key indicators of the epidemic in the country. In another hand, no study has yet explored the extent to which the country could meet the 2030 target. This study aimed to: (i) determine the current level of key indicators of the HIV / AIDS epidemic in the DRC; and (ii) assess whether the DRC could achieve the goal of eliminating HIV / AIDS as a major public health problem by the end of 2030. For the country as a whole and for 24 of its 26 provinces, we performed: (1) a trend analysis of HIV / AIDS surveillance data; and (2) projections of key indicators of the epidemic by 2030 on Spectrum software. In 2017, the DRC is experiencing a generalized epidemic of HIV / AIDS (national prevalence of 1.0\%, 97.5\%CI: $0.85 \%-1.14 \%$ ), which is poorly expansive (national incidence of 6, 97, 5\% IC: 5 - 7 new infections per 10000 person-years of observation). Ten of its 26 provinces have so far experienced a concentrated epidemic. From 2017 to 2030, HIV prevalence will decline for the country as a whole, falling below $1 \%$ by 2018 and reaching $0.76 \%$ by 2030 . The incidence will experience the same overall declining trend. Nine provinces appear to be driving the epidemic. The DRC should target intensively the nine driving provinces of the epidemic and some of its key determinants, in order to fulfill the goal of reducing the HIV / AIDS epidemic to a non-major public health concern by 2030 .
\end{abstract}

Keywords: HIV/AIDS, DRC, Indicators, Projections

\section{Introduction}

The Democratic Republic of the Congo is one of the first countries in the world, and one of the very first in Africa, to have declared the existence of AIDS cases in its hospitals since 1983, and also one of the first to react quickly by setting up comprehensive prevention strategy [1].

High-quality work on understanding the epidemic (modes of transmission), the natural history of the disease, symptoms, opportunistic infections, etc. were led by teams of multinational scientists. Unfortunately, the interruption of the International Cooperation with, and support to, the DRC in the 1990s deprived the country of the necessary resources for the continuation of this important work.

HIV prevalence in DRC seems to be stable, and lower than those of eight of the nine countries with which it shares borders [2].

In terms of etiology, authors have reported the presence of both types of human immunodeficiency virus (HIV1 and HIV2) that are described worldwide. In all cases, these authors described HIV2 infection only in conjunction with HIV1 [3, 4]. However, in a study conducted by Ahuka and collaborators it is argued that only HIV1 would circulate in the DRC, and that the positivity of HIV2 testing would only be cross-reactive. HIV1 is highly diverse in the DRC, which has led some authors to formulate the hypotheses of an older 
and more permanent presence, and even the origin of HIV1 in Central Africa [5-10]. The subtypes A, C, D, G, H and K are always the most frequent, the same strains described by all researches in 20 years, such as Worobey et al [5].

Few appropriate studies have focused on the modes of transmission of HIV infection in the DRC. According to an unpublished survey conducted in 2014: from the 1,475 People Living With HIV (PLWH) who were enrolled from major cities in the country, sex with a partner of the opposite sex remains the most frequent mode of HIV infection $(84.4 \%)$; thereafter, the contamination by transfusion of contaminated blood ( $7.1 \%$ of cases). Ignored for a long time, contamination by sex with a same-sex partner would account for almost $4 \%$ of the total number of cases.

In the DRC, data on HIV / AIDS epidemic indicators across the country are rather limited. The most complete and regular are those obtained during the successive sentinel surveillance activities among pregnant women in Ante Natal Care clinics (ANC). In addition to these, available data include: data from the Prevention of Mother-to-Child Transmission of HIV (PMTCT) service; data from the two Demographic and Health Surveys including an HIV component (DHS +, 2007 and 2013); data collected from projects targeting specific populations (sex workers, youth, uniformed persons, truckers / truck drivers, mineworkers of precious materials, etc.); data produced by the National Program against HIV / AIDS; and data from other specialized Ministry of Health branches and programs with an HIV / AIDS component. The country also held two national science days on HIV / AIDS, which allowed researchers and stakeholders from all walks of life to exchange on various research studies on the subject.

Within this context, the country adopted in 2015 the global vision to end HIV / AIDS as a major public health problem by 2030 . It is therefore urgent to create awareness of the HIV / AIDS epidemic in the DRC, and to assess its chances of achieving this goal. From the literature on the subject, the researchers note that: (a) there has yet a study that provided an overview of the epidemic in the country as a whole and its provinces; (b) reliable methods exist to estimate the prevalence and incidence of HIV infection in a population; but (c) only a few of these processes are applicable to the DRC, with regard to the current state of available data and the technical platform level. We have taken these facts into account by defining the appropriate methodology to make the point about the epidemic in the country and assess its chance of achieving the global target by 2030 .

\section{Methods}

\subsection{Research Design}

We conducted a Surveillance study, to estimate the prevalence, the incidence, and other indicators of HIV/Aids epidemic in the DRC, from pregnant women anonymous and unrelated sentinel surveillance data, collected on a period of 10 years (from 2003 to 2013).

\subsection{Data}

The data analyzed in the current study were obtained from consecutive surveillance activities conducted by the PNLS from 2003 to 2011. These are sociodemographic and behavioral data such as age, marital status, education level, age at first sexual intercourse, age of the current sexual partner; medical and obstetric history such as the number of pregnancies already carried, the number of blood transfusions received; and biological data: syphilis and HIV test results. At each passage, a minimum of 385 pregnant women are tested at each surveillance site according to the national surveillance protocol. As this protocol was modified from 2013 onwards, we resubmitted all the 17,211 samples collected that year to an HIV testing according to the old algorithm, thus providing additional data to those already existing.

\subsection{Procedures}

For the country as a whole and for 24 of its 26 provinces, we performed: (a) a trend analysis of HIV / AIDS surveillance data in sentinel sites among pregnant women attending antenatal clinics; and (b) Spectrum projections of prevalence, incidence, number of people living with HIV, AIDS-related deaths, and number of pregnant women requiring prevention of transmission of the virus to their children [11].

To estimate HIV prevalence and its trend from the ANC surveillance date, we considered the whole group of pregnant women tested by passage, whereas for the incidence we used the prevalence of the group of women aged 15 to 19 and carrying their first pregnancy, those HIV-positive having been considered as incidental cases, and all of these women tested as exposed for the year. The chi-square homogeneity test was used to assess differences between annual prevalences / incidences. The Cochrane-Armitage and Mantel tests were used to search for a trend of these parameters over time [12]. Serial correlation of residues was also measured. For cases where this correlation was positive, the Cochrane-Orcutt procedure was preferred to CochraneArmitage in the trend assessment and in drawing the regression line. The mean change per unit of the score was calculated, which is the annual rate of increase or decrease in prevalence / incidence. This average change is expressed as a percentage, as are the values of its confidence interval.

The NCSS software (version 11) and the DESCRIBE program (version 2.33) of the WINPEPI software were used for these analyzes. Some graphs have been made on Stata (version 13), or Microsoft Excel. Spectrum modeling (the AIM model) incorporated both demographic data, sentinel HIV surveillance data, and other relevant studies in the field (including the two Demographic and Health Surveys in the country), to determine the level of the epidemic in the country and / or province and to estimate what it would be in 2030, using a double-least squares estimation equation [13]. These projections were possible for 22 of the country's 26 provinces, but not for the 4 that had less than 3 passages of 
ANC based surveillance activities by 2013 . The possible association between HIV serology and any of the factors collected during the passage of surveillance activities was assessed by the adjusted odds ratio, through a logistic regression of HIV serology on those potential factors.

\section{Results}

Analyzes of surveillance data included a total of 109,553 pregnant women surveyed and tested for HIV in the country since the start of surveillance in 2003, until 2013. Between 2003 and 2013, HIV prevalence among pregnant women accused a gradually increasing pace $(\mathrm{p}=0.00031)$ with a slope of +0.101 and an average annual increase of $2.34 \%$. The incidence estimated as the prevalence in the group of women under 20 years of age and carrying their first pregnancy showed no significant trend during this time $(\mathrm{p}=$ 0.266).

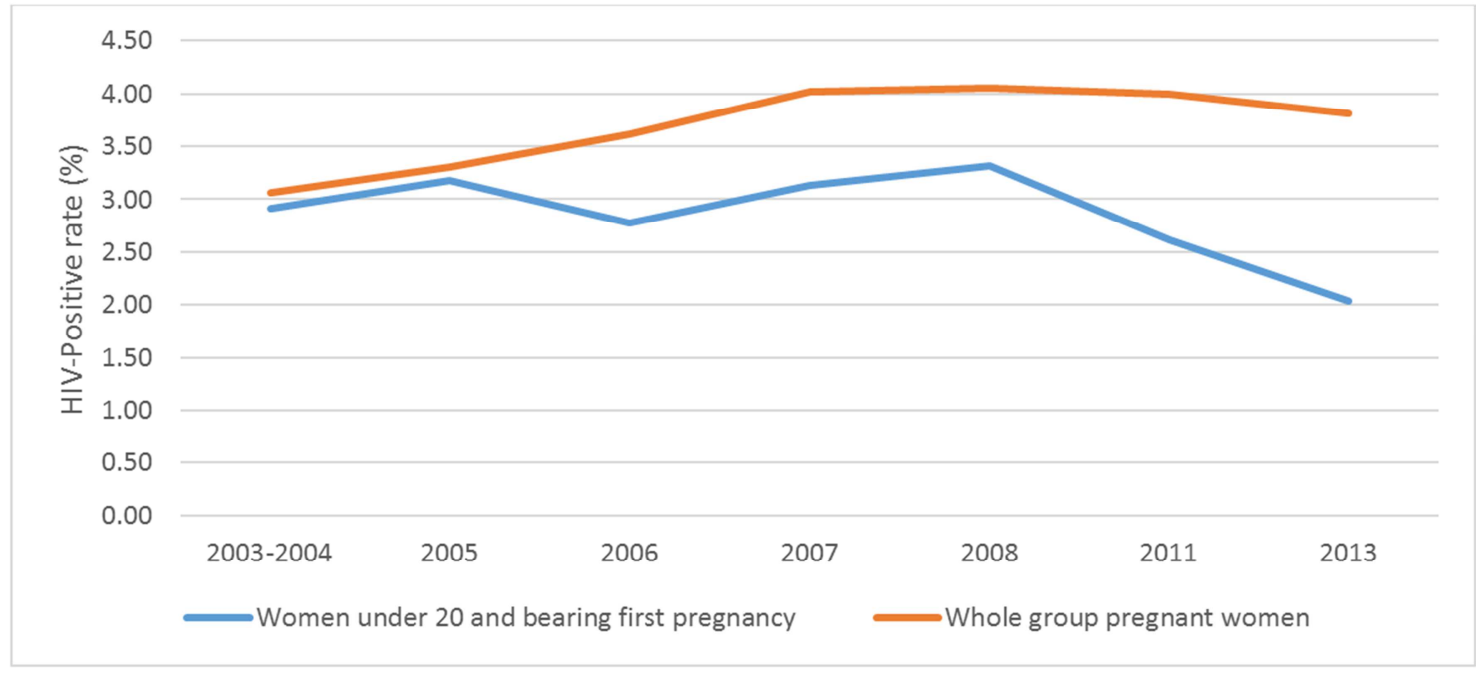

Figure 1. Changes in HIV prevalence among pregnant women in the DRC from 2003 to 2013.

In 2017 , the country has been experiencing a generalized epidemic (national prevalence of $1.0 \%, 97.5 \% \mathrm{CI}: 0.85 \%-$ $1.14 \%$ ), and not very expansive (national incidence of 6 , 97.5\%CI 5-7 new infections per 10,000 person-years of observation). From a total of 455,878 people living with HIV in the DRC during 2017, eighty-four percent of them are eligible for ARV treatment on the basis of the country's current criteria. Moreover, Table 1 shows that during 2017, nearly 30,000 estimated pregnant women might require an HIV prevention service for their babies in the DRC.

Table 1. Some figures summarizing the HIV / AIDS epidemic in the DRC in 2017, estimates and $97.5 \%$ CI.

\begin{tabular}{llll}
\hline People Living with HIV Adults and Children & & Annual Aids-related death among 15+ & 7522 \\
\hline Estimate & 455787 & Estimate & $(6052-8863)$ \\
$97.5 \% \mathrm{CI}$ & $(391239-528629)$ & $97.5 \% \mathrm{CI}$ & 384902 \\
Annual number of new HIV infections & & Needs for ARV therapy for 15+ & $(330143-447056)$ \\
Estimate & 24219 & Estimate & \\
$97.5 \% \mathrm{CI}$ & $(19859-29286)$ & $97.5 \% \mathrm{CI}$ & 19392 \\
Annual number of deaths due to AIDS (total) & & Needs for ARV therapy among children & $(16808-22326)$ \\
Estimate & 9644 & Estimate & \\
$97.5 \% \mathrm{CI}$ & $(7939-11308)$ & $97.5 \% \mathrm{CI}$ & 28049 \\
HIV Prevalence (15-49) \% & & Mothers needing PMTCT \\
Estimate & 1 & Estimate & $(23707-32731)$ \\
$97.5 \% \mathrm{CI}$ & $(0.85-1.14)$ & $97.5 \% \mathrm{CI}$ & 227889 \\
HIV infection incidence (15-49) \% & & AIDS orphans & $(183544-270140)$ \\
Estimate & 0.06 & Estimate & $97.5 \% \mathrm{CI}$ \\
$97.5 \% \mathrm{CI}$ & $(0.05-0.07)$ & & \\
\hline
\end{tabular}

Regional estimates and projections were possible for 22 of the country's 26 provinces. Table 2 shows that in nine of these provinces the prevalence is consistently greater than
$1 \%$. Moreover, all these provinces have an HIV incidence greater than one new infection per 1000 person-years of observation. 
Table 2. Prevalence and incidence of HIV in 22 provinces of the DRC in 2017, estimates and 97.5\%IC.

\begin{tabular}{lll}
\hline \multirow{2}{*}{ Province } & Prevalence $\mathbf{( p . ~ 1 0 0 )}$ & Incidence (p. 100) \\
\cline { 2 - 3 } & Estimate $\mathbf{( 9 7 . 5 \%} \mathbf{C I})$ & Estimate (97.5\% CI) \\
\hline Haut Uele & $6.98(6.25-7.36)$ & $0.57(0.50-0.63)$ \\
Maniema & $3.44(1.16-6.57)$ & $0.30(0.08-0.56)$ \\
Ituri & $3.33(1.07-7.01)$ & $0.29(0.09-0.54)$ \\
Lomami & $2.34(2.06-2.73)$ & $0.29(0.21-0.39)$ \\
Bas Uele & $2.22(1.80-2.87)$ & $0.16(0.09-0.35)$ \\
Sankuru & $1.72(1.47-1.85)$ & $0.13(0.09-0.15)$ \\
Kasaï Oriental & $1.69(0.76-2.82)$ & $0.28(0.09-0.52)$ \\
Kinshasa & $1.58(1.05-2.04)$ & $0.11(0.07-0.15)$ \\
Tanganyika & $1.53(1.11-1.96)$ & $0.11(0.04-0.18)$ \\
Haut Katanga & $1.44(0.93-2.11)$ & $0.10(0.06-0.16)$ \\
Kasai & $1.42(0.74-2.55)$ & $0.32(0.11-0.79)$ \\
Mongala & $1.07(0.70-2.00)$ & $0.18(0.08-0.49)$ \\
Nord Kivu & $0.81(0.36-1.31)$ & $0.03(0.01-0.07)$ \\
Equateur & $0.64(0.30-1.54)$ & $0.04(0.01-0.22)$ \\
Nord Ubangi & $0.55(0.34-0.79)$ & $0.01(0.01-0.34)$ \\
Haut Lomami & $0.50(0.23-0.83)$ & $0.01(0.00-0.02)$ \\
Kasai Central & $0.49(0.33-0.88)$ & $0.01(0.01-0.03)$ \\
Tshopo & $0.35(0.32-0.41)$ & $0.03(0.02-0.05)$ \\
Sud Kivu & $0.32(0.14-0.67)$ & $0.01(0.00-0.02)$ \\
Kwilu & $0.26(0.09-0.55)$ & $0.01(0.00-0.03)$ \\
Maï Ndombe & $0.23(0.06-0.62)$ & $0.01(0.00-0.03)$ \\
Kongo Central & $0.17(0.08-0.36)$ & $0.01(0.00-0.02)$ \\
\hline
\end{tabular}

The projections predict a progressive decrease in the prevalence of HIV in the adult population of DRC. By 2018, this prevalence falls below $1 \%$, characteristic of a concentrated epidemic, and will continue to decline to $0.76 \%$ (97.5\% CI $0.55 \%$ - $1.11 \%$ ) by 2030 , while the incidence decreases from $0.06 \%$, i.e. six new infections per 10000 person-years of observations, in 2017 to $0.04 \%$ in 2020 . Then, from 2022, the maximalist estimates of the annual incidence will again be approaching pre-2017 levels. This increase is more marked from 2025, and continues until 2030.

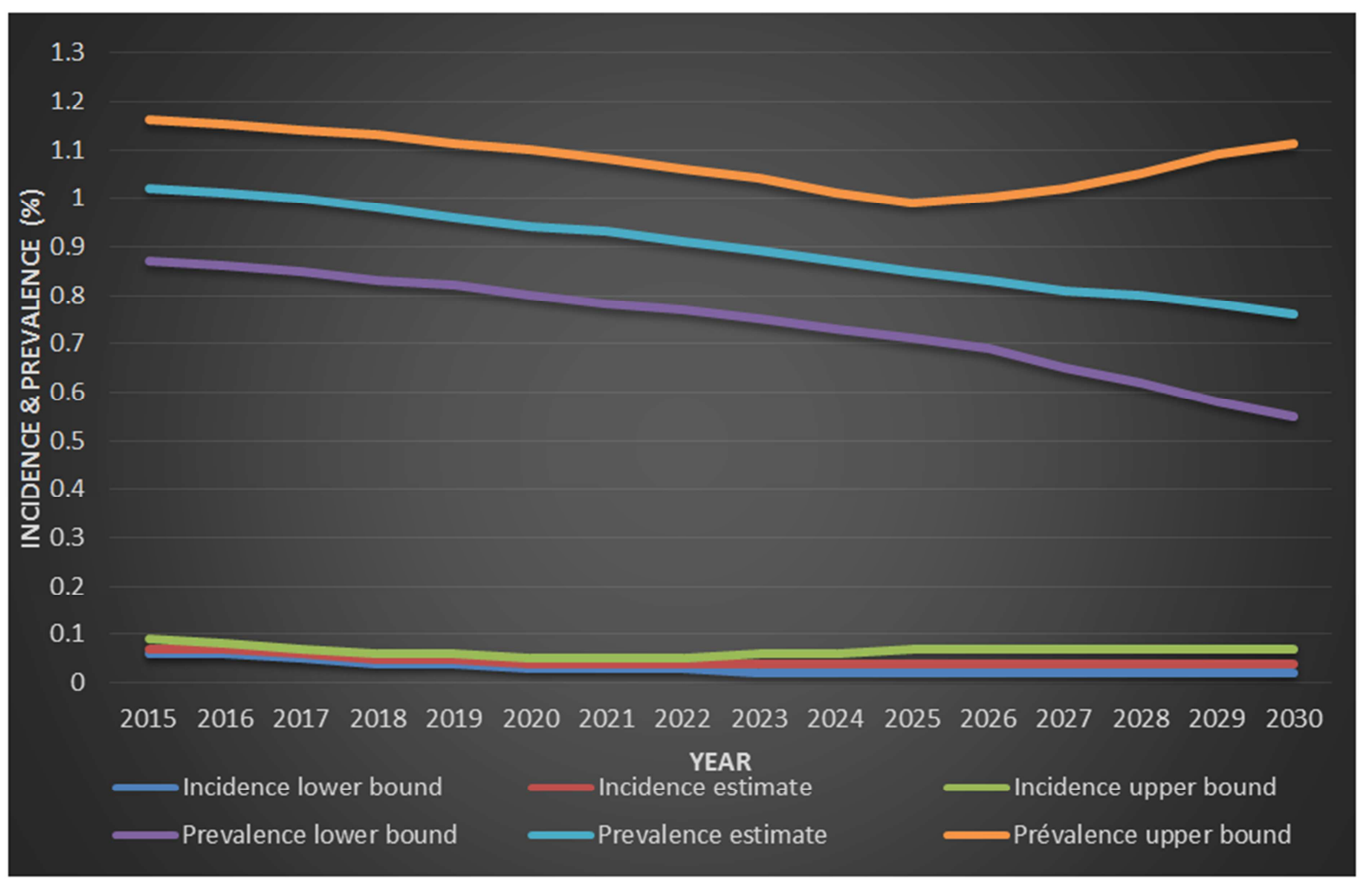

Figure 2. Projected prevalence and incidence of HIV infection in the adult population of the DRC, 2015-2030: Estimates, lower and upper 97.5\%CI bounds. 
In terms of other epidemic indicators for 2030: the number of PLWH will be close to 600,000, of whom more than $80 \%$ will be eligible for ARV treatment; that of new infections around 25,000 per year; and annual AIDS deaths in adults at 4,500.

Table 3. Some figures summarizing the HIV / AIDS epidemic in the DRC in 2030, estimates and 97.5\%CI.

\begin{tabular}{|c|c|c|c|}
\hline People Living with HIV Adults and Children & & Annual Aids-related death among $15+$ & \\
\hline Estimate & 583226 & Estimate & 4496 \\
\hline $97.5 \% \mathrm{CI}$ & $(430684-828432)$ & $97.5 \% \mathrm{CI}$ & $(3563-5789)$ \\
\hline Annual number of new HIV infections & & Needs for ARV therapy for $15+$ & \\
\hline Estimate & 24427 & Estimate & 541820 \\
\hline $97.5 \% \mathrm{CI}$ & $(14137-40670)$ & $97.5 \% \mathrm{CI}$ & $(401818-765494)$ \\
\hline Annual number of deaths due to AIDS (total) & & Needs for ARV therapy among children & \\
\hline Estimate & 5648 & Estimate & 8791 \\
\hline $97.5 \% \mathrm{CI}$ & $(4519$ - 7329$)$ & $97.5 \% \mathrm{CI}$ & $(6521-12198)$ \\
\hline HIV Prevalence (15-49) \% & & Mothers needing PMTCT & \\
\hline Estimate & 0.76 & Estimate & 27013 \\
\hline $97.5 \% \mathrm{CI}$ & $(0.55-1.11)$ & $97.5 \% \mathrm{CI}$ & $(18620-39871)$ \\
\hline HIV infection incidence (15-49) \% & & AIDS orphans & \\
\hline Estimate & 0.04 & Estimate & 59193 \\
\hline $97.5 \% \mathrm{CI}$ & $(0.05-0.07)$ & $97.5 \% \mathrm{CI}$ & $(48423-70323)$ \\
\hline
\end{tabular}

At the decentralized level, four provinces will still record a prevalence equal to or greater than $5 \%$, eight provinces a prevalence of between $1 \%$ and $5 \%$, and ten provinces a concentrated epidemic (less than $1 \%$ prevalence). For four of the 26 provinces in the country, it was not possible to make the projections because of insufficient current data available. (Figure 3)

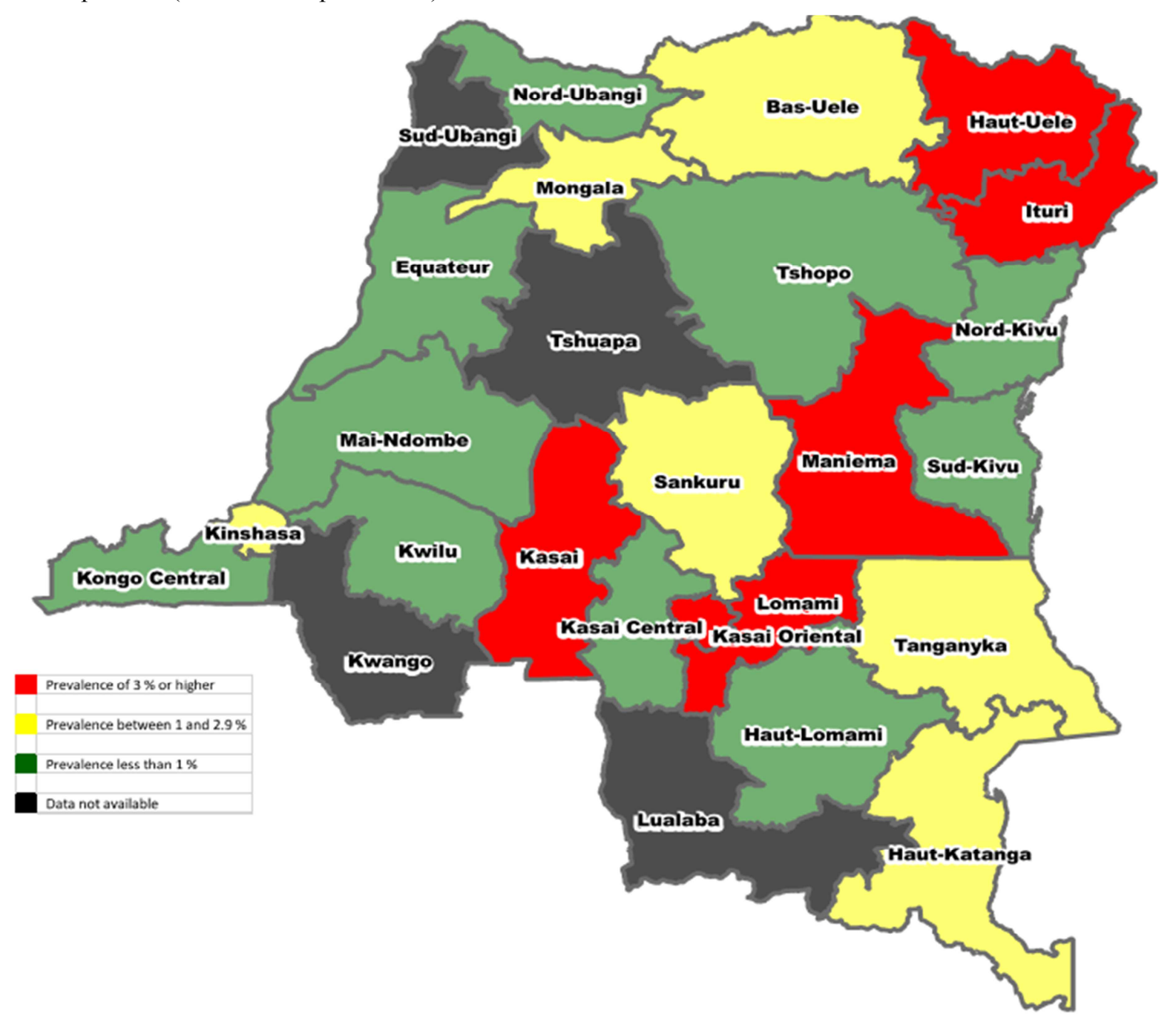

Figure 3. HIV prevalence in 22 provinces of DRC, projected for 2030. 


\section{Discussion}

This research was conducted to assess the current situation of HIV / AIDS in the Democratic Republic of Congo; and the extent in to which the country may or may not achieve the goal set by communities and nations for 2030 to eliminate HIV / AIDS as a major public health problem. This is the first research that has tackled the current epidemiological profile of HIV / AIDS in 22 of 26 provinces in the DRC, in addition to that of the country itself. Its main limitation: the use of the prevalence and estimation of the incidence of HIV among pregnant women to estimate those of the general population, given the context of predominantly sexual transmission of the infection. To approximate the general population as closely as possible, we used as incidence estimator the prevalence among women coming to ANC sentinel sites, aged younger than 20 years and carrying their first pregnancy, corrected by a factor of 0.8 . On the other hand, the use of Spectrum software helped to take into account other behavioral and population data.

Magnitude:

The DRC is currently experiencing a generalized HIV / AIDS epidemic (national median prevalence of $1.0 \%, 97.5 \%$ CI: $0.85 \%-1.14 \%$ ). The current incidence of HIV infection in the country is six new infections per 10,000 person-years of observation. The number of people living with HIV is 455,787 (97.5\% CI: 391,239 - 528,629), of whom 404,294 are eligible for antiretroviral treatment on the basis of the current National HIV / AIDS Program criteria. The prevalence found here is in line with those found by other studies in the general population in the DRC, including the Demographic and Health Survey coupled with HIV testing (DHS + ), that found an overall HIV prevalence of $1.2 \%$ for the year 2013 [14]. Estimates of the Joint United Nations Program on HIV / AIDS, UNAIDS, give similar figures ( $1.1 \%$ in $2013 ; 0.8 \% 97.5 \%$ CI $0.7 \%-1.1 \%$ in 2015$)$. If the 2015 figures remained unchanged until 2017, the national prevalence in our study would not be any different from those of UNAIDS. From the nine countries bordering the DRC, only one (Burundi: 1.0\% 95\% CI: $0.8 \%$ - 1.3\%) has such low HIV prevalence. It seems therefore that the recognition of the epidemic early, and establishing a coherent framework of struggle, has contributed to halting the spread of HIV in the DRC and that the epidemic is on the way of concentration [15]. According to our estimates, the number of PLWH currently in the DRC, is 455,787 (97.5\% CI: 391,239 - 528,629). During 2013, Granich and colleagues estimated the number for PLWH at 440,000 (95\% CI: 370,000 - 520000 ) [16]. UNAIDS estimated this number (for 2015) at 370000 (95\% CI: 290000 - 460 000). All these figures show that our estimates, which are more accurate and have incorporated a greater number of parameters, corroborate other sources. The figures produced to characterize the HIV epidemic in the DRC to date are therefore valid and can be used as such. In terms of incidence, our estimates and projections resulted in $6(97.5 \%$
CI: 5-7) new infections per 10,000 person-years of observation in 2017. Direct measurement of incidence of $\mathrm{HIV}$ in the general population is both costly and ethically questionable. Alternatives were proposed to overcome this difficulty: monitoring incidence in specific, well-defined groups; estimating incidence from prevalence data in the general population; estimation from pregnancy monitoring data; or measurement by indirect biological methods, such as the back-calculation method; or by searching for biological markers of a recent infection [17-23]. Each of these alternatives has its own limitations. In this study, we estimated the incidence of HIV for the period before 2014 by two methods: the use of prevalence in pregnant women aged 15 to 19 and bearing their first pregnancy, corrected by the factor 0.8; and the use of Spectrum software for estimates and projections. For the period after 2014 (due to the fact that the sentinel surveillance data are no longer usable due to the change of the test algorithm), only the projection figures on Spectrum have been reported. Given that the median age at first sexual intercourse is 16 years of age, and that by the end of their 18 th year $85 \%$ of women in this series had had their first sexual intercourse, we preferred to consider ages 15 to 19 , rather than 15 to 25 years of age, recommended by UNAIDS. Moreover, taking into account the incidence of HIV infection on fertility, we retained women who had their first pregnancy (presuming that they had just started their sexual activity). Finally, we applied the UNAIDS recommended correction factor of 0.8 [24-26]. In spite of all this refining, we found an annual incidence of $2 \%$ during 2013 (the last year for which we have HIV sentinel surveillance data using the same screening algorithm since 2003), i.e. 200 new infections per 10000 person-years of observation. This figure seems high, confirming that the estimation of the incidence of HIV in the adult population on the basis of prevalence data among pregnant women has non negligible limitations [26]. Indeed, when the HIV epidemic reaches maturity, the majority of women living with HIV are at an advanced stage of infection, characterized by a decline in fertility [27]. On the other hand, an HIV epidemic lasting more than 20 years (like that of the DRC) would ultimately result in higher infection rates in adult populations, many of whom are post-menopausal women. However, Zaba et al found that the prevalence of HIV among pregnant women aged 15-24 and the incidence of HIV infection in the general population are correlated, as are their trends over time [20]. The second method (estimation and projection on Spectrum) gives the annual incidence scope of $0.08 \%$ (eight new infections per 10,000 person-years of observation) for 2013. This figure is 25 times lower than that found on the basis of prevalence among young pregnant women, but it seems more valid for the DRC [28]. Indeed, based on the prevalence of consecutive population surveys, Hallett et al estimated the incidence of HIV in the general population at $0.34 \%$ in Tanzania and $1.12 \%$ in Zambia [19]. In Uganda's rural districts, this incidence was estimated to be $0.5 \%$ in 2004 and $0.8 \%$ in $2007[29,30]$. The rate of $0.08 \%$ is therefore adapted 
to the situation of the DRC, which has an HIV prevalence of at least three times lower than each of these three countries (approximately 12 times less for Zambia).

At the provincial level: the prevalence of the HIV epidemic is at different levels in the provinces of the DRC. In 2017, only one province has a prevalence above 5\% (Haut Uele [ $6.8 \%$ ]); eleven others have a prevalence below $5 \%$ but greater than 1\% (Maniema [3.44\%], Ituri [3.33\%], Lomami $[2.34 \%]$, Low Uele [2.22\%], Sankuru [1.72\%], Kasaï Oriental [1.69\%], Kinshasa [1.58\%], Tanganyika [1.53\%], Haut Katanga [1.44\%], Kasaï [1.42\%] and Mongala $[1.07 \%])$; and the others have a prevalence of less than $1 \%$, the lowest being the Province of Kongo Central [0.17\%]. To our knowledge, there are no other studies that have measured HIV prevalence in the general population of the current 26 provinces of the DRC. Our results will therefore be compared with those of the only population survey that measured this prevalence when there were only 11 provinces in the DRC: DHS 2103 - 2014. It produced provincial prevalences of HIV in these 11 provinces, which was a first in the DRC. Five of these eleven provinces had not been dismembered: Bas Congo, Kinshasa, Maniema, North Kivu, and South Kivu. For Bas Congo, since renamed as Kongo Central, its HIV prevalence measured at $0.2 \%$ by the DHS (2013). Our study results in exactly the same prevalence for the year 2013: $0.2 \%(97.5 \%$ CI: $0.09 \%-0.37 \%)$. In the absence of a more recent population survey, we can therefore confidently use the figures from our estimates and projections and claim that the current HIV prevalence in the general adult population of Kongo Central province is $0.17 \%$ (97.5\% CI: $0.08 \%$ $0.36 \%$ ). This is the lowest provincial prevalence of HIV in the DRC. Taking into account some (unpublished) programmatic data on HIV prevalence in some population sub-groups (sex workers, blood donors, etc.) that increases from $1.36 \%$ to $7.9 \%$, we can classify the current HIV / AIDS epidemic in the Kongo Central province at the concentrated level. The capital city of Kinshasa is a metropolis with more than 10 million inhabitants. The DHS estimated the prevalence of HIV in this city at $1.6 \%$ in 2013 . Moreover, our estimates and projections for that same year provide the figure of $1.54 \%(97.5 \% \mathrm{CI}: 1.03 \%-2.01 \%)$. Here again, it is with confidence that we can use the current figures given by our estimates and projections, i.e. in 2017 a prevalence of $1.58 \%(97.5 \% \mathrm{CI}: 1.05 \%-2,04 \%)$, showing that this city currently has a generalized HIV / AIDS epidemic. At the time when there were only 11 provinces in the DRC, Maniema was the province with the highest HIV prevalence (4\%): DHS 2013-2014. Our methodology results reflect similar figures during 2013: $3.1 \%$ (97.5\% CI: $1.12 \%$ $5.82 \%$ ). In 2017 , this province continues to have a generalized epidemic with a prevalence of $3.44 \%(97.5 \% \mathrm{CI}$ : $1.16 \%-6.57 \%$ ). Programmatic data show a low deployment of basic prevention and care services in this province, which could explain this prevalence. In North Kivu, the HIV prevalence measured by the DHS was $0.9 \%$ in 2013. Our estimates and projections put this prevalence for the same year at $0.98 \%(97.5 \%$ CI: $0.42 \%-1.54 \%)$. For South Kivu,
HIV prevalence in the general adult population from our estimates and projections for $2013(0.44 \%$, IC97.5\% CI: $0.16 \%-0.92 \%)$ were no different from those found by the DHS for the same year $(0.4 \%)$. Currently, the prevalence of $\mathrm{HIV}$ in this province is $0.32 \%(97.5 \% \mathrm{CI}: 0.14 \%-0.67 \%)$. South Kivu is therefore experiencing a concentrated HIV / AIDS epidemic. For the rest of the provinces, the prevalence of HIV varies in the same proportions as those of the five.

The incidence of HIV varies greatly from one province to another in the DRC. The researchers found that from 22 provinces in the DRC for which data were available, the incidence during 2017 is less than 10 new infections per 10,000 person-years of observation in 10 provinces. This low level of incidence indicates a low level of HIV transmission among the general population. However, 11 provinces have both high prevalences (above 1.5\%) and high incidence (more than 10 new infections per 10000 person-years of observation) of HIV infection: Haut Uele, Kasai, Maniema, Ituri, Lomami, Kasai Oriental, Mongala, Bas Uele, Sankuru, Kinshasa and Tanganyika. These 11 provinces are thus the drivers of the HIV epidemic in the country, and should be the priority of investment in the fight. Three of the 11 provinces (Kasai Oriental, Lomami, and Sankuru) originate from the former diamond-producing province of Kasai Oriental, and other two (Haut Uele and Bas Uele) from the former Province Orientale, rich in small-scale gold mining.

Trends and forecasts:

The world community has set the objective to end the HIV epidemic as a major problem of public health by 2030 . The projection of key indicators of the epidemic (prevalence and incidence) carried out in this study makes a prediction as to what would happen in the DRC, if control settings (scale of funding, coverage of interventions and their scaling, etc.) were to maintain their current pace. Nationally, from 2017 to 2030, HIV prevalence will decline in the DRC, falling below $1 \%$ by 2018 and reaching $0.76 \%$ by 2030 . It could be said that the epidemic will then be concentrated and thus that the DRC will have succeeded in reducing HIV / AIDS to a less expressive level than a major public health problem. However, the maximalist estimate, the upper bound of the $97.5 \%$ confidence interval, of this prevalence would still give a figure including $1 \%(97.5 \% \mathrm{CI}: 0.55 \%-1.11 \%)$. To achieve this goal with confidence in 2030, the DRC must not only continue to build on the momentum but also increase high-impact interventions (such as scaling up ARV treatment and targeting high-risk populations and as the hinge populations). At the provincial level, the projection over time of HIV prevalence in the provinces of the DRC will reflect different rates and different levels by 2030: six in twenty-two provinces (Ituri, Kasai, Kasai Oriental, Lomami, Maniema and Mongala) will experience an increasing prevalence from 2017 to 2030; a decreasing prevalence for five in twenty-two provinces (Kasaï Central, Kwilu, Maï Ndombe, North Kivu and South Kivu); and an unchanging prevalence for the remaining eleven provinces. Finally, by 2030, twelve provinces in the DRC will still have a generalized epidemic, and the remaining ten will have a concentrated one. 


\section{Conclusion}

From 2017 to 2030, the prevalence and incidence of HIV in the general population of the DRC will experience a progressive and sustained decline. By 2030, the median prevalence of the country will be $0.8 \%$, characteristic of a concentrated epidemic. However, twelve provinces of the 26 in the country will still record a generalized epidemic in 2030, although a bit expansive. If it maintains the current momentum of the struggle, focusing intensively on the nine motor provinces of the epidemic and some of its key determinants, the DRC could reach 2030 with a decline in the HIV/AIDS epidemic to a level of a non-major public health problem.

\section{References}

[1] JM M, Francis H, TC Q, et al. Hiv seroprevalence among hospital workers in Kinshasa, Zaire: Lack of association with occupational exposure. JAMA [Internet]. 1986 Dec 12;256(22):3099-102.

[2] Mulanga-Kabeya C, Nzilambi N, Edidi B, Minlangu M, Tshimpaka T, Kambembo L, et al. Evidence of stable HIV seroprevalences in selected populations in the Democratic Republic of the Congo. AIDS. 1998;12(8):905-10.

[3] Yang C, Dash B, Hanna SL, Frances HS, Nzilambi N, Colebunders RC, et al. Predominance of HIV type 1 subtype $\mathrm{G}$ among commercial sex workers from Kinshasa, Democratic Republic of Congo. AIDS Res Hum Retroviruses. 2001;17(4):361-5.

[4] Mokili JL, Rogers M, Carr JK, Simmonds P, Bopopi JM, Foley BT, et al. Identification of a novel clade of human immunodeficiency virus type 1 in Democratic Republic of Congo. AIDS Res Hum Retroviruses. 2002;18(11):817-23.

[5] Worobey M, Gemmel M, Teuwen DE, Haselkorn T, Bunce M, Muyembe J, et al. Direct Evidence of Extensive Diversity of HIV-1 in Kinshasa by 1960. 2013;455(7213):661-4.

[6] Vidal N, Bazepeo SE, Mulanga C, Delaporte E, Peeters M. Genetic characterization of eight full-length HIV type 1 genomes from the Democratic Republic of Congo (DRC) reveal a new subsubtype, $\mathrm{A} 5$, in the $\mathrm{A}$ radiation that predominates in the recombinant structure of CRF26_A5U. AIDS Res Hum Retroviruses. 2009;25(8):823-32.

[7] Yang C, Li M, Mokili JLK, Winter J, Lubaki NM, Mwandagalirwa KM, et al. Genetic diversification and recombination of HIV type 1 group $M$ in Kinshasa, Democratic Republic of Congo. AIDS Res Hum Retroviruses. 2005;21(7):661-6.

[8] Faria NR, Rambaut A, Suchard MA, Baele G, Bedford T, Ward MJ, et al. The early spread and epidemic ignition of HIV-1 in human populations. 2015;346(6205):56-61.

[9] Vidal N, Peeters M, Mulanga-Kabeya C, Nzilambi N, Robertson D, Ilunga W, et al. Unprecedented Degree of Human Immunodeficiency Virus Type 1 (HIV-1) Group M Genetic Diversity in the Democratic Republic of Congo Suggests that the HIV-1 Pandemic Originated in Central Africa. J Virol. 2000;74(22):10498-507.
[10] Rodgers MA, Wilkinson E, Vallari A, McArthur C, Sthreshley L, Brennan CA, et al. Sensitive Next-Generation Sequencing Method Reveals Deep Genetic Diversity of HIV-1 in the Democratic Republic of the Congo. J Virol [Internet]. 2017;91(6):1-18.

[11] Stover J, Johnson P, Zaba B, Zwahlen M, Dabis F, Ekpini RE. The Spectrum projection package: improvements in estimating mortality, ART needs, PMTCT impact and uncertainty bounds The Spectrum projection package: improvements in estimating mortality, ART needs, PMTCT impact and uncertainty bounds; 2008

[12] Abramson JH. Abramson, J. H. WINPEPI updated: computer programs for epidemiologists, and their teaching potential. Epidemiologic Perspectives \& Innovations; 2011.

[13] Bao L. A new infectious disease model for estimating and projecting HIV/AIDS epidemics. Sex Transm Infect [Internet]. 2012;88:158-64.

[14] MPSMRM M. Enquête Démographique et de Santé en République Démocratique du Congo 2013-2014. Rockville, Maryland, USA : MPSMRM, MSP et ICF International; 2014.

[15] Cuadros DF, Awad SF, Abu-Raddad LJ. Mapping HIV clustering: a strategy for identifying populations at high risk of HIV infection in sub-Saharan Africa. Int $J$ Health Geogr [Internet]. International Journal of Health Geographics; 2013;12(1):28.

[16] Granich R, Gupta S, Hersh B, Williams B, Montaner J, Young $\mathrm{B}$, et al. Trends in AIDS deaths, new infections and ART coverage in the top 30 countries with the highest AIDS mortality burden; 1990-2013. PLoS One. 2015;10(7):1-16.

[17] Chow EPF, Wilson DP, Zhang L. Estimating HIV incidence among female partners of bisexual men in China. Int J Infect Dis. International Society for Infectious Diseases; 2012 May;16(5):e312-20.

[18] Cleghorn FR, Jack N, Murphy JR, Edwards J, Mahabir B, Paul R, et al. Direct and indirect estimates of HIV-1 incidence in a high-prevalence population. Am $\mathrm{J}$ Epidemiol. 1998;147(9):834-9.

[19] Hallett TB, Stover J, Mishra V, Ghys PD, Gregson S. Europe PMC Funders Group Estimates of HIV incidence from household-based prevalence surveys. 2013;24(1):147-52.

[20] Zaba B, Boerma T, White R. Monitoring the AIDS epidemic using HIV prevalence data among young women attending antenatal clinics: prospects and problems. AIDS. 2000 Jul;14(11):1633-45.

[21] R JC, Becker NG. Estimating HIV incidence using dates of both HIV and AIDS diagnoses. 2000;(July 1999):1165-77.

[22] Remis RS, Palmer RWH. Testing bias in calculating HIV incidence from the Serologic Testing Algorithm for Recent HIV Seroconversion. AIDS. 2009 Mar;23(4):493-503.

[23] Brookmeyer R. Should biomarker estimates of HIV incidence be adjusted ? 2009;(December 2008):485-91.

[24] Gouws E, Mishra V, Fowler TB. Comparison of adult HIV prevalence from national population-based surveys and antenatal clinic surveillance in countries with generalised epidemics: implications for calibrating surveillance data. Sex Transm Infect. 2008 Aug;84 Suppl 1:i17-23. 
[25] UNAIDS. Rapport ONUSIDA sur l'épidémie mondiale du Sida, 2011. Geneva; 2011.

[26] UNAIDS \& WHO. Aids Epidemic Update: December 2007. Geneva; 2007.

[27] Brookmeyer R. Measuring the HIV/AIDS epidemic: Approaches and challenges. Epidemiol Rev. 2010;32(1):2637.

[28] Adilo TM, Wordofa HM. Prevalence of fertility desire and its associated factors among 15 - to 49 -year-old people living with HIV / AIDS in Addis Ababa, Ethiopia : a cross-sectional study design. HIV/AIDS - Res Palliat Care. 2017;167-76.
[29] Shafer LA, Biraro S, Nakiyingi-Miiro J, Kamali A, Ssematimba D, Ouma J, et al. HIV prevalence and incidence are no longer falling in southwest Uganda: evidence from a rural population cohort 1989-2005. AIDS. 2008 Aug;22(13):1641-9.

[30] Kim A, Hallett T, Stover J, Gouws E, Musinguzi J, Mureithi PK, et al. Estimating HIV incidence among adults in Kenya and Uganda: a systematic comparison of multiple methods. PLoS One [Internet]. 2011 Jan [cited 2013 Mar 17]; 6(3):e17535. 